\title{
Propuesta de contenidos en plan de capacitación administrativa para emprendimientos en Quito
}

Fecha de recepción: 2021-11-05 • Fecha de aceptación: 2022-01-14 • Fecha de publicación: 2022-02-10

Carlos José Arias Ronquillo ${ }^{1}$

Universidad Tecnológica Israel, Ecuador

carias@uisrael.edu.ec

https://orcid.org/0000-0002-4672-5168

Carolina Paola Jaramillo Bayas ${ }^{2}$

Universidad Tecnológica Israel, Ecuador

cjaramillo@uisrael.edu.ec

https://orcid.org/0000-0001-9856-9892

Lorena Elizabeth Muquis Tituaña ${ }^{3}$

Universidad Tecnológica Israel, Ecuador

Imuquis@uisrael.edu.ec

https://orcid.org/0000-0002-3823-9530

\section{RESUMEN}

La academia, dentro del entono de vinculación con la sociedad, se ve cada vez más enlazada con establecer formas de apoyo a los sectores vulnerables. En este caso se trata de los emprendedores del Distrito Metropolitano de Quito, pues la actividad empresarial presenta problemas al iniciar el negocio y luego al comercializar sus productos. Existe incertidumbre, porque en los emprendimientos, las necesidades económicas que se presentan ayudan a tomar decisiones erróneas sobre el manejo de su negocio, ya que el escaso conocimiento en algunos temas administrativos y comerciales puede llevar al declive del emprendedor.

El presente trabajo de investigación plantea como objetivo diseñar una malla curricular con contenidos temáticos, requeridos por los emprendedores en el área administrativa y comercial, dentro de un plan de capacitación, para lo cual se aplicó una metodología cuantitativa, basada en 
el criterio de expertos de instituciones involucradas en procesos de capacitación a emprendedores, así como con base en los resultados de investigaciones previas realizadas dentro de la propia universidad, y de cuyos resultados se pudo establecer las áreas temáticas administrativa, comercial y financiera contable, cuyos principales contenidos se organizaron en tres niveles en un plan de capacitaciones para emprendimientos, establecidos en el presente estudio.

\title{
PALABRAS CLAVE: capacitación, emprendimiento, academia, sectores vulnerables
}

\begin{abstract}
The academy, in its relationship with society, is increasingly involved in establishing forms of support for vulnerable sectors. In this case, we are talking about entrepreneurs in the Metropolitan District of Quito, because the business activity presents problems when starting the business and then marketing their products. There is uncertainty, because in entrepreneurship, the economic needs that arise help to make wrong decisions about the management of their business, since the lack of knowledge in some administrative and commercial issues can lead to the decline of the entrepreneur.

The objective of this research work is to design a curriculum with thematic contents required by entrepreneurs in the administrative and commercial area, within a training plan, for which a quantitative methodology was applied, based on the criteria of experts from institutions involved in training processes for entrepreneurs, and based on the results of previous research carried out within the university itself, and from whose results it was possible to establish the administrative, commercial and financial-accounting thematic areas, whose main contents were organized into three levels in a training plan for entrepreneurship, established in this st.
\end{abstract}

KEYWORDS: training, entrepreneurship, academia, vulnerable sectors 


\section{Introducción}

En investigaciones anteriores, como el Análisis de las necesidades de capacitación administrativa y comercial de los emprendimientos. Caso de aplicación en la Universidad Tecnológica Israel (Arias et al., 2021), mencionan la importancia de que las Instituciones de Educación Superior (IES) "no solo son entes generadores de conocimiento, sino además como una de las instituciones de la sociedad llamadas a contribuir justamente al desarrollo local de las comunidades microempresariales nacionales, sobre todo dentro de su área de influencia".

Actualmente, atravesamos por una pandemia mundial conocida como el COVID - 19, la cual ha cambiado la vida normal de las personas y en la que se desarrollaban a diario. Adicionalmente, la misma ha venido acompañada con una crisis económica agresiva que ha afectado a distintos sectores económicos del país.

Por estas razones, muchos emprendedores se han visto amenazados con la nueva realidad del país, para lo cual necesitan conocimientos técnicos en temas administrativos, financieros, ventas y bioseguridad, que les permita reactivar sus negocios y poder adaptarse de mejor manera a la nueva "normalidad" con mejores herramientas y protocolos de bioseguridad, mejorando así su participación en el mercado, incrementando sus ventas a través de un servicio de calidad, y sobre todo con las normas de bioseguridad necesarias para mantener un negocio seguro y confiable para sus clientes.

En el 2018, en la provincia de Pichincha, de acuerdo al tamaño de las empresas por niveles de ventas, las microempresas representan el $79 \%$ y las pymes el $15 \%$. Las empresariales y corporativas solo representan el $4 \%$ y $2 \%$, respectivamente, como se observa en la Tabla 1 . El comportamiento de la participación por tipo de empresas de la provincia de Pichincha es similar al del Ecuador, y sobre todo la mayor parte de microempresas se encuentran en la ciudad de Quito.

\section{Tabla 1.}

Composición del tipo de empresas en el Ecuador

\begin{tabular}{lc}
\hline \multicolumn{1}{c}{ Tipo de empresa } & Participación \\
\hline Microempresas (Ventas entre \$0 y \$1.000.000) & $78,68 \%$ \\
\hline $\begin{array}{l}\text { Pequeñas y medianas (PYMES) (Ventas entre \$1.000.000 - } \\
\$ 5.000 .000)\end{array}$ & $14,84 \%$ \\
\hline Empresariales (Ventas entre \$5.000.000 y \$20.000.000) & $4,39 \%$ \\
\hline Corporativas (Ventas mayores a \$20.000.000) & $2,09 \%$ \\
\hline
\end{tabular}

Fuente: Superintendencia de Compañías, Valores y Seguros del Ecuador (2018)

Tomando en consideración esta alta participación en el sector económico del país, las IES, por medio de la docencia, la investigación y la vinculación con la comunidad, trabajando conjuntamente con los estudiantes, se ha logrado determinar las fortalezas y debilidades en el campo administrativo y de comercialización, por medio de las entrevistas y encuestas 
desarrolladas en las investigaciones anteriores se puede tener una visión clara sobre los temas más relevantes que forman parte de la propuesta de contenidos para brindar las capacitaciones.

Se debe además reflexionar que los negocios y micro, pequeñas y medianas empresas (MiPymes) de la ciudad presentan algunas falencias, una de las más importantes es la necesidad de capacitación de los comerciantes y empresarios en las distintas áreas de la empresa. Esta situación se presenta debido a que los comerciantes y empresarios carecen de conocimientos técnicos sobre administración, mercadeo y ventas, finanzas, contabilidad, recursos humanos, producción, tecnologías de información, etc.; los cuales no permiten una eficiencia en los procesos y ocasionan una disminución de la productividad, que a larga genera falta de competitividad a los emprendedores (González et al., 2011).

Además, esto se refleja debido a los cambios en el entorno, a la actualización de las herramientas tecnológicas disponibles para el respectivo funcionamiento, las nuevas normas legales y tributarias, las nuevas formas de motivación de los colaboradores, los nuevos competidores, la elaboración de una plataforma estratégica efectiva, etc.; lo que hace que los comerciantes y empresarios requieran capacitaciones en las diferentes áreas o temas de interés, con el propósito de tomar las mejores decisiones con base en información confiable y veraz, y poder llevar una buena administración de sus negocios.

En lo referente a la capacitación para emprendimientos por parte de las universidades en el Ecuador, se debe mencionar que el desarrollo de las actividades empresariales de los emprendedores pone en evidencia la investigación que deben realizar, tanto docentes, como estudiantes, para ajustar los diversos modelos teóricos a las exigencias prácticas de las comunidades emprendedoras y sus negocios que los requieren para su operación diaria. Así mismo, es necesario un proceso de seguimiento y evaluación de los cursos de capacitación de corto alcance, realizados con la finalidad de medir su impacto y resultados en las comunidades donde se han llevado a cabo, y de esta forma tomar decisiones de mantener o reorientar las temáticas de los proyectos de formación ejecutados, de forma que logren su sostenibilidad en el tiempo.

Las capacitaciones deben cumplir con temas específicos, tales como la determinación de costos y gastos operativos del negocio, los requisitos de su formación legal, el levantamiento de los principales procesos operativos del negocio, los canales de comercialización y promoción, sus principales fuentes de financiamiento disponibles, entre otros, que ayuden a los emprendedores a lograr los objetivos mencionados, para ello es necesario que las instituciones al frente de estos programas de capacitación conozcan cuáles son las necesidades de formación que demandan estos grupos de personas, muchas de las cuales ya han iniciado el camino del emprendimiento con sus pequeñas unidades económicas y productivas, las cuales requieren ser apoyadas en diferentes ámbitos de acción.

Es por estas y muchas razones que cumplir con un plan de capacitación organizado y desarrollado, de acuerdo a las necesidades de los emprendedores, facilitará la mejor captación 
de los conocimientos; porque es sabido que si alguna persona se encuentra con desconocimiento en finanzas, otra tal vez la puede tener en costos y a su vez otra en tecnologías, por ende, es importante conocer de acuerdo a las investigaciones previas realizadas como el de Arias et al. (2021), cuáles son los conocimientos previos y falencias en cada emprendedor, y con ello cumplir con la organización en temas de interés colectivo que aporten al emprendedor y el manejo de su negocio, satisfaciendo las necesidades de los mismos. En ese sentido, el presente trabajo de investigación plantea como objetivo diseñar una malla curricular con contenidos temáticos requeridos por los emprendedores en el área administrativa y comercial, dentro de un plan de capacitación.

Para la selección de los temas y contenidos de un plan de capacitación para emprendedores se debe comprender primero lo que es un emprendimiento, entendido "desde la formación emprendedora como un conjunto de competencias que parte de la actitud de la persona para captar oportunidades de su entorno, lo cual requiere un pensamiento sistémico, convertirlas en ideas innovadoras con base a su creatividad y generar proyectos productivos en diferentes campos para beneficio propio y de su entorno" (Uribe Macías, 2013)

A comienzos del siglo XX, Jean Baptista Say -uno de los pioneros de la ciencia económicageneralizaba el alcance de la palabra emprendedor, conocida en su acepción francesa como entrepreneur, a toda aquella persona capaz de dirigir una empresa, actuando como intermediario entre el capital y el trabajo, incorporando la idea del riesgo: "el empresario está expuesto a todos los riesgos mientras se aprovecha de todo lo que pueda serle favorable" (Drair et al., 2013).

Según Lederman \& Messina (2014), los emprendedores de éxito son individuos que transforman ideas en iniciativas rentables. A menudo, esta transformación requiere talentos especiales, como la capacidad de innovar, introducir nuevos productos y explorar otros mercados. Se trata de un proceso que también precisa la habilidad de dirigir a otras personas, priorizar las tareas para aumentar la eficiencia productiva y darles a los recursos disponibles el mejor uso posible.

Como ejemplos prácticos de emprendedores en marcha que han requerido formarse para mejorar sus competencias y sus negocios para mantenerse en el mercado, son los comerciantes de la Asociación Chimborazo del Centro Comercial la Bahía de Tumbaco en Quito, quienes requirieron capacitaciones en temas como servicio al cliente, relaciones humanas y contabilidad básica, entre otros aspectos, o los comerciantes del Comité Central de la Loma Grande de Quito, quienes también requirieron capacitarse en temas como investigaciones de mercado y contabilidad básica de sus negocios, necesidades que fueron cubiertas por parte de estudiantes y docentes del la Universidad Tecnológica Israel (mencionada como UISRAEL en adelante).

\section{Metodología}

Para el estudio de la propuesta de contenidos de un plan de capacitación administrativa y comercial se utilizó un enfoque cuantitativo, que permitió analizar la realidad particular de las necesidades suscitadas en el medio, de forma objetiva, a través de resultados comprobables, proveniente de la aplicación de instrumentos o técnicas como las entrevistas a funcionarios de las entidades que participan en los proyectos de vinculación con centros de educación superior, 
como la Universidad Tecnológica Israel. Aparte, se consideraron los resultados previos obtenidos y analizados de las encuestas de necesidades de capacitación aplicados a los emprendedores participantes de los proyectos de capacitación con la universidad y otros estudios preliminares de carácter exploratorio, realizados por la misma institución, dentro de los proyectos de capacitación dirigidos a emprendedores de la zona.

Para la aplicación de las entrevistas se aplicó el método Delphi de consulta a expertos en el área, a través de un cuestionario revisado y validado por un equipo de docentes especialistas en varias áreas de la administración, el marketing y las finanzas de la UISRAEL, que trabajan además en proyectos de capacitación y fue delimitado con dieciséis (16) preguntas, de tipo abiertas para los expertos, generadas en función del enfoque de los contenidos que debería mantener un plan de capacitación administrativo y comercial para emprendedores.

Los siete expertos que participaron en las entrevistas pertenecen a las siguientes instituciones:

- Ministerio de la Producción, Comercio Exterior, Inversiones y Pesca del Ecuador

- Ministerio de Inclusión Económica y Social del Ecuador

- Fundación Heifer Ecuador

- Corporación CEASFRON

- Gobierno Autónomo descentralizado de Calderón, Quito

- Comité Central de la Loma Grande, Quito

- Programa ITAP Rural, de la Fundación a Favor de la Vida

A los expertos se les consultó con preguntas abiertas sobre las características socio económicas, niveles de instrucción sugeridos para procesos de capacitación, principales actividades económicas que desarrollan los emprendedores, qué tipo de problemas presentan los emprendedores y cuáles son sus competencias, áreas de conocimiento que requieren capacitación, así como temáticas de capacitación de acuerdo a la realidad actual, cuáles son las estrategias para conseguir resultados efectivos durante el proceso. De las respuestas abiertas obtenidas de las entrevistas se las resumió en una matriz con los aspectos más importantes mencionados, y luego se tomaron las ideas principales para resumir las características más importantes mencionadas de cada aspecto consultado. Por tanto, de estas fuentes obtenidas en el presente trabajo sirvieron para definir cuáles deberían ser los contenidos que debería contener un plan de capacitación en los temas administrativos y comerciales dirigido a emprendedores.

\section{Resultados}

Como producto de los cuestionamientos realizados a los expertos de las entidades participantes en los proyectos de capacitación para emprendedores, se pudieron extraer las siguientes conclusiones más importantes, mismas que serán compaginadas con los resultados que se obtuvieron de las encuestas aplicadas a ochenta y ocho emprendedores, previamente realizadas y publicadas en la investigación de Arias et al. (2021), con la finalidad de establecer 
una programación y contenidos a tratar dentro de un plan de capacitación estructurado para emprendedores.

Al cuestionamiento realizado a los expertos entrevistados sobre de las características socioeconómicas más importantes, respecto de los emprendedores con los que trabaja la institución, respondieron un $80 \%$ de los entrevistados que los emprendedores pertenecen a clases socioeconómicas medias y bajas, generalmente pequeños productores artesanales pertenecientes a sectores vulnerables de la sociedad, donde predominan actividades con bajos niveles de innovación, con accesos limitados a la tecnología, los cuales pueden mejorar en los sectores más jóvenes de la población.

En relación a los niveles de instrucción que presentan los emprendedores con los que trabajan, expresaron que es variado, predominando el nivel bajo de instrucción o educación con un aproximado de un 35\% de los emprendedores que tiene un nivel básico de escolaridad, seguido por un $30 \%$ con nivel de bachillerato secundario, un 30\% con estudios de tercer nivel y un $5 \%$ con estudios de cuarto nivel, porcentajes que varían para cada entidad, pero donde predominan los bajos niveles de educación formal en la mayoría de emprendedores, como se muestra en la Figura 1.

\section{Figura 1.}

Niveles de instrucción

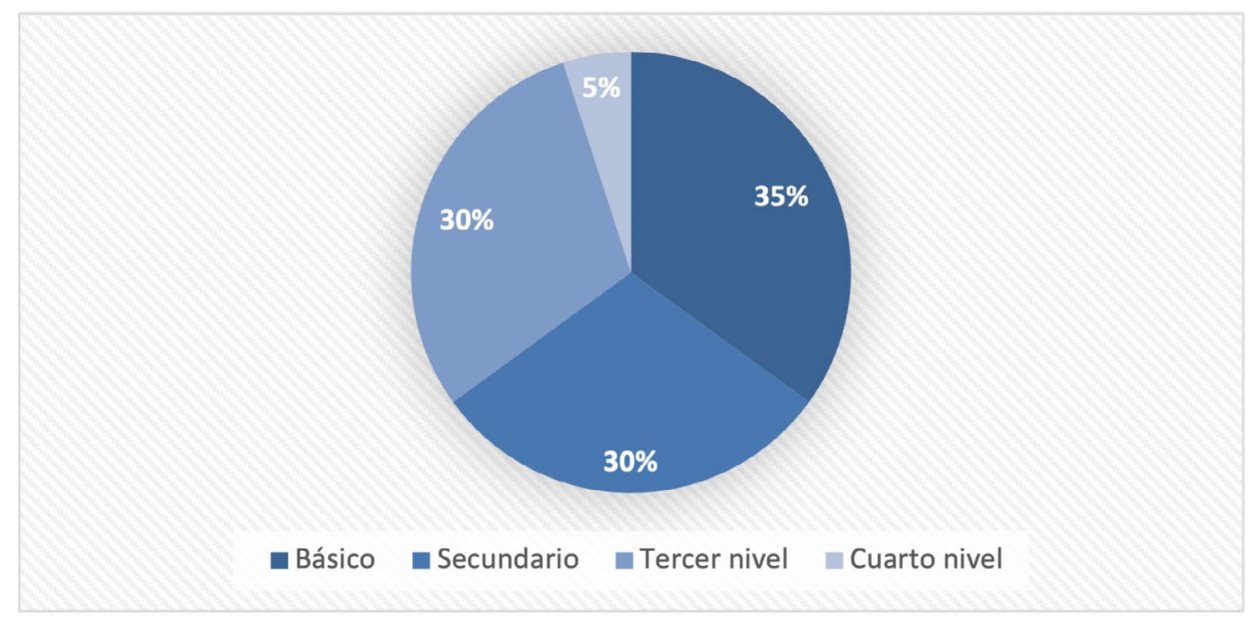

Sobre las principales áreas de actividades económicas a las que se dedican los emprendedores, indicaron que básicamente se dedican a actividades como la producción agrícola, comercio al por menor (en algunos casos ambulantes) de alimentos, artículos de bazar y limpieza, preparación de alimentos, fabricación de textiles, muebles y artesanías, crianza de animales, entre las más importantes.

Los principales problemas que han identificado en los emprendedores con los que trabajan las instituciones, los encuestados manifestaron entre los principales el desconocimiento sobre temas como la formalización de un emprendimiento en cuanto a documentos y requisitos de 
funcionamiento, las alternativas de financiamiento, el mercado de sus productos y clientes, así como de la competencia, la falta de educación o capacitación para manejar sus emprendimientos, pues muchos negocios se heredan de familiares, la falta de planificación de actividades, los escasos o limitados flujos de dinero que poseen para manejar sus negocios, entre los principales.

En referencia al tipo de competencias que tienen los emprendedores que trabajan con las instituciones, manifestaron que, si bien en su mayoría cuentan con competencias laborales muy elementales, como conocimientos productivos empíricos y ancestrales, trabajo en equipo, organización y atención al cliente básica, entre otros, pueden destacar competencias personales como la resistencia o perseverancia, fortaleza física y mental, solidaridad, intuición para buscar mercados, desempeño práctico, iniciativa y actitud para el trabajo basado en conocimientos empíricos y ancestrales.

Respecto al tipo de ayuda que buscan los emprendedores frente a sus competencias, las instituciones indicaron que requieren apoyo de diversos tipos, desde indicaciones para las preparaciones de empaques para sus productos, información sobre fuentes de financiamiento, el aprovisionamiento de materias primas, los diseños de sus marcas, hasta capacitación para el manejo económico, contable y legal.

Cuando se les consultó sobre las áreas de conocimiento que consideran que los emprendedores necesitan capacitarse, opinaron que el área financiera con énfasis en determinación de costos, márgenes de utilidad y fijación de precios, manejo contable y tributario del negocio, el área comercial con énfasis en la promoción, a través del comercio y marketing electrónico y atención al cliente; los aspectos legales para la creación y formalización del negocio, los regímenes laborales e inclusive el manejo de aspectos emocionales y de salud personal.

En referencia al cuestionamiento de las temáticas que han sido las más importantes para los emprendedores registrados al momento de capacitarse, refieren a los temas de promoción a través de herramientas digitales del comercio electrónico, elaboración del plan de negocios para reestructurar su microproyecto de inversión, manejo contable, financiero de costos y tributario del negocio, servicio al cliente, y ciertos temas específicos de tipo técnico productivo de cada negocio en menor grado.

Para las razones por las cuales creen que los emprendedores buscan ser capacitados, expresaron algunas opiniones, entre ellas la búsqueda de información sobre alternativas para comercializar sus productos en los mercados nacionales e internacionales, formas de incrementar sus ingresos por ventas, requisitos para acceder a fuentes de financiamiento, manejo contable y financiero de sus negocios, formas de manejar o gestionar administrativamente sus negocios y en ocasiones para obtener un certificado de capacitación que necesitan como requisito para acceder a una fuente de financiamiento específica.

Al cuestionamiento sobre las preferencias de los emprendedores en cuanto a la forma de ser capacitados respecto a horarios, lugares, métodos de enseñanza, niveles de cursos, entre otros, expresaron que prefieren capacitaciones de duraciones cortas, de tipo personalizadas, en entornos de confianza y diálogo personal, en base a aspectos prácticos del negocio, antes que teóricos, 
con contenidos y material de tipo visual o interactivo que contengan texto, audio y video, durante tiempos cortos, con preferencia de fines de semana, donde disponen algo más de tiempo, a sus labores diarias, en horarios de la tarde o noche, donde sus actividades bajan de intensidad, y de preferencia en lugares cercanos a sus negocios.

Sobre las características que deberían tener los capacitadores de los cursos para emprendedores, los encuestados opinaron que deben tener un conocimiento de tipo práctico que se apoye en el aprendizaje por resolución de problemas, y en los principios de la andragogía con técnicas y herramientas participativas que ello implica, que posean conocimientos sobre los temas a desarrollar en los campos administrativos, financieros y comerciales, que sean buenos comunicadores, sensibles, empáticos, activos y dinámicos.

Al preguntarles sobre el tipo de material de apoyo que creen serviría para mejorar los procesos de capacitación de los emprendedores, comentaron como idea principal que sean visuales, permanentes y cortos, que pueden ser escritos, para que los mantengan como información posterior a la capacitación, en las aulas físicas o virtual, puede ser material interactivo.

Recomiendan además textos cortos con material audiovisual y didáctico que faciliten la interacción.

Y en cuanto al tipo de contenido que debería incluirse en los cursos de capacitación, indicaron que deben ser en primer término contenidos básicos para el manejo de una pequeña unidad empresarial, donde se incluyan temas legales o de normativa básica, de la administración de recursos y comercialización, que despierten la creatividad e innovación, ayuden a establecer y minimizar los riesgos de los negocios, y que promuevan la superación personal y profesional a través de la ejecución de proyectos con la aplicación de casos prácticos.

Finalmente, en referencia a las sugerencias de mejoras que podrían recomendar para la realización de capacitaciones para emprendedores, expresaron que este tipo de capacitaciones deben ser prácticos, con técnicas interactivas y participativas con utilización de técnicas como los juegos, aplicando la teoría a la práctica, desarrollados en un entorno de confianza y que traten de abarcar a los diversos sectores económico-productivos de la sociedad.

Tomando en consideración los resultados expuestos en la presente investigación, a los que se adicionan los resultados del estudio denominado Análisis de las necesidades de capacitación administrativa y comercial de los emprendimientos. Caso de aplicación en la Universidad Tecnológica Israel (Arias et al., 2020), donde se aplicó una encuesta a ochenta y ocho emprendedores de la ciudad de Quito, entre los principales resultados se pudo determinar, entre otros aspectos, lo siguiente.

Dentro de los contenidos de capacitación en las áreas administrativas y comerciales que están demandando los emprendedores para la gestión de sus negocios se debe dar especial atención a temas comerciales o de marketing, como estrategias competitivas del mercado, marketing digital, la innovación productiva, administrativos como el liderazgo empresarial, el control de procesos y de manejo financiero-contable dentro de los emprendimientos, pues requieren apoyo para el levantamiento y análisis de la información financiera de sus negocios, tema que es necesario se articulen en una malla curricular con niveles de aprobación. 
El grupo de trabajo investigativo desarrolló una malla curricular de contenidos, que con base en la información recabada de emprendedores que poseen conocimientos básicos, hasta emprendedores que poseen conocimientos medios y en menor grado conocimientos más avanzados del manejo de sus negocios, se organizó la estructura de un plan de capacitación en tres niveles de enseñanza:

- Nivel básico

- Nivel medio

- Nivel avanzado

Cabe mencionar que los niveles y contenidos propuestos están direccionados hacia emprendimientos que ya funcionen en la práctica, independientemente su tiempo de funcionamiento, para que el emprendedor en función de su nivel de conocimiento pueda ingresar al nivel de su formación; sin embargo, se puede considerar que aquellos emprendedores que se encuentran en una etapa de idea del negocio no establecido aun, puedan iniciar en el nivel básico y avanzar a los siguientes niveles.

Dentro de cada nivel se consideran, a su vez, tres campos principales del conocimiento, en función de los requerimientos encontrados:

- Área de administración o gestión general del negocio

- Área de comercialización del negocio

- Área financiera y contable del negocio

Dentro del área de administración se revisará con el emprendedor los siguientes contenidos en cada nivel:

- En el nivel básico se enseñará a delimitar su filosofía empresarial, establecer su misión y visión, establecer sus objetivos empresariales y realizar un diagnóstico situacional general con el análisis interno y externo de su unidad empresarial.

- En el nivel medio se tratará sobre la elaboración de los planes de acción y objetivos de corto plazo del emprendimiento, se realizará un análisis de fortalezas, oportunidades y amenazas del negocio, la valoración de aspectos de tamaño y localización del negocio, los requisitos básicos de formación empresarial en el país, y aspectos de innovación empresarial.

- En el nivel avanzado se les ayudará a crear y seleccionar estrategias empresariales, armar los organigramas funcionales básicos de la empresa, la organización del personal y a levantar los principales procesos administrativos del negocio.

Dentro del área comercial y de marketing se revisará con el emprendedor los siguientes aspectos: 
- En el nivel básico se revisarán los aspectos del producto, bien o servicio a producir y vender, sus características y empaques, aspectos del consumidor o cliente, la publicidad y promoción y los canales de comercialización.

- En el nivel medio se tratará sobre la elaboración de investigaciones de mercado básicas para determinar la oferta y demanda de sus productos, lo que le permita establecer la demanda insatisfecha del bien o servicio y concluir con la elaboración de los planes de ventas del negocio.

- En el nivel avanzado se les impartirá temas como las estrategias comerciales, las ruedas de negocios para presentar productos, las alianzas comerciales, el uso del comercio electrónico y medios digitales para comercializar sus bienes o servicios y el presupuesto de marketing en los negocios.

Dentro del área financiera y contable se revisará lo siguiente:

- En el nivel básico se revisará los aspectos de los egresos, identificando costos y gastos totales del negocio, así como sus ingresos para la determinación de precios y márgenes de ganancias, aspectos del ahorro y la inversión, los principales documentos comerciales utilizados y su registro en la contabilidad básica, hasta la determinación de los activos, pasivos y patrimonio del negocio.

- En el nivel medio se tratará sobre los informes financieros básicos del negocio a través de los estados de resultados, de situación financiera y flujos de caja, además, se revisarán las obligaciones tributarias y laborales, así como las principales fuentes de financiamiento con sus costos.

- En el nivel avanzado se tratarán temas como el punto de equilibrio del negocio, las proyecciones financieras a través de presupuestos básicos, un análisis financiero básico del negocio a través de indicadores claves, el riesgo financiero y una evaluación básica de proyectos de inversión.

Cabe mencionar que, con base en las preferencias de los emprendedores y la opinión de los expertos, los temas de capacitación se han considerado para una duración de 30 horas de capacitación por cada nivel de cada área, es decir, que el programa completo cubriría unas 270 horas de capacitación distribuidas en el tiempo.

El programa de contenidos expuestos se resume en la siguiente Tabla 2, que muestra en detalle los temas propuestos con los objetivos por cada nivel y área, para el plan de capacitación para emprendedores. 


\section{Tabla 2.}

Propuesta de malla curricular de contenidos para un plan de capacitación para emprendedores en el Distrito Metropolitano de Quito

\begin{tabular}{|c|c|c|c|}
\hline Nivel & $\begin{array}{c}\text { Área de } \\
\text { conocimiento }\end{array}$ & Objetivo del nivel & Temas de contenidos propuestos \\
\hline \multirow[t]{15}{*}{ Nivel básico } & \multirow[t]{4}{*}{ Administración } & \multirow{4}{*}{$\begin{array}{c}\text { Mostrar al emprendedor } \\
\text { la filosofía empresarial } \\
\text { y el diagnóstico de la } \\
\text { empresa }\end{array}$} & La filosofía empresarial \\
\hline & & & Los objetivos empresariales a corto plazo \\
\hline & & & Análisis interno de la empresa \\
\hline & & & Análisis externo de la empresa \\
\hline & \multirow{5}{*}{$\begin{array}{l}\text { Comercial y de } \\
\text { marketing }\end{array}$} & \multirow{5}{*}{$\begin{array}{c}\text { Introducir al } \\
\text { emprendedor en los } \\
\text { conceptos básicos del } \\
\text { marketing para sus } \\
\text { negocios }\end{array}$} & Definición del producto \\
\hline & & & Empaques del producto \\
\hline & & & El consumidor o cliente \\
\hline & & & La promoción y publicidad \\
\hline & & & Los canales de comercialización \\
\hline & \multirow{6}{*}{$\begin{array}{l}\text { Finanzas } \\
\text { ycontabilidad }\end{array}$} & \multirow{6}{*}{$\begin{array}{c}\text { Introducir al } \\
\text { emprendedor en los } \\
\text { conceptos iniciales } \\
\text { de las finanzas y la } \\
\text { contabilidad }\end{array}$} & Los egresos: los costos y gastos \\
\hline & & & Los ingresos y el precio y margen de ganancias \\
\hline & & & El ahorro y la inversión \\
\hline & & & Los documentos comerciales \\
\hline & & & Los registros contables y financieros básicos \\
\hline & & & Los activos, pasivos y el patrimonio \\
\hline \multirow[t]{15}{*}{ Nivel medio } & \multirow{5}{*}{ Administración } & \multirow{5}{*}{$\begin{array}{l}\text { Mostrar al emprendedor } \\
\text { la capacidad de crear } \\
\text { objetivos a mediano y } \\
\text { largo plazo. }\end{array}$} & $\begin{array}{l}\text { Objetivos a corto y mediano plazo en los planes } \\
\text { de acción }\end{array}$ \\
\hline & & & Realizar el análisis FODA \\
\hline & & & Aspectos del tamaño y localización del negocio \\
\hline & & & Requisitos de formación empresarial \\
\hline & & & Innovación empresarial \\
\hline & \multirow{5}{*}{$\begin{array}{l}\text { Comercial y de } \\
\text { marketing }\end{array}$} & \multirow{5}{*}{$\begin{array}{c}\text { Mostrar al emprendedor } \\
\text { técnicas para realizar } \\
\text { una investigación de } \\
\text { mercados }\end{array}$} & La investigación de mercados \\
\hline & & & La demanda total \\
\hline & & & La oferta o competencia \\
\hline & & & La demanda insatisfecha \\
\hline & & & El plan de ventas \\
\hline & \multirow{5}{*}{$\begin{array}{l}\text { Finanzas } \\
\text { y contabilidad }\end{array}$} & \multirow{5}{*}{$\begin{array}{c}\text { Mostrar al emprendedor } \\
\text { los reportes financiero- } \\
\text { contables básicos } \\
\text { del negocio, sus } \\
\text { obligaciones tributarias } \\
\text { con el estado y } \\
\text { laborales del recurso } \\
\text { humano }\end{array}$} & Los estados financieros básicos \\
\hline & & & $\begin{array}{l}\text { Estados de resultados, de Situación y Flujos de } \\
\text { caja del negocio }\end{array}$ \\
\hline & & & $\begin{array}{l}\text { Las fuentes de financiamiento } \\
\text { empresarial }\end{array}$ \\
\hline & & & Las obligaciones tributarias \\
\hline & & & Las obligaciones laborales \\
\hline
\end{tabular}




\begin{tabular}{|c|c|c|c|}
\hline \multirow[t]{13}{*}{ Nivel avanzado } & \multirow[t]{4}{*}{ Administración } & \multirow{4}{*}{$\begin{array}{c}\text { Analizar y seleccionar } \\
\text { estrategias } \\
\text { empresariales a utilizar } \\
\text { en los negocios }\end{array}$} & Selección de estrategias \\
\hline & & & Organigrama funcional de la empresa \\
\hline & & & Procesos administrativos del negocio \\
\hline & & & Organización del personal o recurso humano \\
\hline & \multirow{5}{*}{$\begin{array}{l}\text { Comercial y de } \\
\text { marketing }\end{array}$} & \multirow{5}{*}{$\begin{array}{c}\text { Mostrar al emprendedor } \\
\text { estrategias de } \\
\text { marketing y } \\
\text { herramientas digitales } \\
\text { para mejorar la } \\
\text { comercialización de sus } \\
\text { productos }\end{array}$} & Las estrategias comerciales \\
\hline & & & Ruedas de negocios \\
\hline & & & El presupuesto de marketing \\
\hline & & & El comercio electrónico \\
\hline & & & Marketing digital \\
\hline & \multirow{4}{*}{$\begin{array}{l}\text { Finanzas } \\
\text { ycontabilidad }\end{array}$} & \multirow{4}{*}{$\begin{array}{c}\text { Mostrar al emprendedor } \\
\text { formas de evaluar } \\
\text { financieramente a su } \\
\text { negocio }\end{array}$} & El punto de equilibrio del negocio \\
\hline & & & $\begin{array}{l}\text { Proyecciones financieras a través de } \\
\text { presupuestos }\end{array}$ \\
\hline & & & El análisis financiero básico del negocio \\
\hline & & & El riesgo financiero empresarial \\
\hline
\end{tabular}

La evaluación de los proyectos de inversión.

\section{Conclusiones}

Como conclusiones del estudio se pudo determinar que los contenidos de capacitaciones para emprendimientos en áreas administrativas, comerciales y financieras que requieren los emprendedores son de diversa índole, específicamente con énfasis primero en el conocimiento básico de los conceptos de manejo de una empresa, tales como la planificación, filosofía empresarial de una compañía, costos, ingresos y márgenes de utilidad a obtener, y también conceptos comerciales del marketing, producto, marca, y luego de aspectos prácticos que deben combinarse, como los requisitos básicos de funcionamiento y formalización del negocio, las obligaciones tributarias, las formas de promoción de sus productos, entre otras, aspectos que son esenciales para el funcionamiento de un emprendimiento, y que se han considerado en la propuesta de contenidos del plan de capacitación elaborado.

Los temas presentados en los campos administrativos, comerciales y financieros deben ser organizados en tres niveles, con base en el grado de conocimiento del emprendedor en estos temas, que depende de su formación académica, la cual es variada, desde emprendedores con educación básica, hasta aquellos que poseen una formación académica de tercer o cuarto nivel; así se plantea un nivel básico, que introduce al emprendedor en los conceptos administrativos básicos de formación del negocio, un nivel medio que le ayuda a comprender herramientas técnicas básicas para la administración del negocio, y un nivel avanzado que le enseña a establecer estrategias para competir en el mercado y a evaluar sus resultados obtenidos, por lo que es importante que se dividan los temas en dichos niveles y se capaciten a los emprendedores, conforme vayan asimilando los conocimientos.

Respecto de las metodologías o formas de enfoque que deben aplicarse para la enseñanza de los temas propuestos a los emprendedores, es importante recalcar que los mismos deben ser 
más prácticos, que incluyan actividades de aplicación de los temas vistos a cada uno de sus emprendimientos.

Los programas de capacitación como el propuesto deben ser de corto alcance en el tiempo, por las propias disponibilidades de tiempos operativos de los emprendedores, pero sostenidos y constantes, apoyados por materiales didácticos que sean específicos y atractivos para los capacitados y que cuenten con un seguimiento o evaluación posterior a su desarrollo para comprobar su efectividad, lo que en todo caso evidencia que la instrucción o capacitación es necesaria dentro de un proceso de profesionalización de los emprendedores, que ayude a disminuir sus riesgos de operación y les brinde una oportunidad de sobrevivir y permanecer en el tiempo. 


\section{Referencias}

Arias, C., Ibarra, F., Tulcán, A., \& Córdova, M. (2021). Análisis de las necesidades de capacitación administrativa y comercial de los emprendimientos. Caso de aplicación en la Universidad Tecnológica Israel. En U. T. Israel, Gestión Administrativa de las organizaciones, actualidad y perspectivas, pp. 285-301. Universidad Tecnológica Israel.

Baena Paz, G. (2014). Metodología de la Investigación. Grupo Editorial Patria.

Chiavenato, I. (2007). Administración de recursos humanos. Mc Graw-Hill.

Corona Treviño, L. (2021). Emprendimientos, financiamiento y legislación. Ed. Miguel Ángel Porrúa.

Draier, E., Huarte, J., Lebendiker, A., \& Mendez, A. (2013). Claves para emprendedores. Buenos Aires: s/e.

González Cano, M., Mendoza Austria, F., Jiménez Alvarado, M., \& Villegas González, E. (2014). Problema de competitividad: la capacitación. https://www.uaeh.edu.mx/investigacion/productos/4854/problema de competitivdad.pdf

Hernández Blázquez, B. (2001). Técnicas estadísticas de investigación social. Ediciones Díaz de Santos S.A.

Lederman, D., \& Messina, J. (2014). El emprendimiento en América Latina, muchas empresas y poca innovación. Banco Mundial.

Montaño Gallo, J. (2016). Proyecto de plan de capacitación para los comerciantes y empresarios matriculados y/o afiliados a la Cámara de Comercio de Villavicencio, con el objetivo de ser competitivos. [Tesis de grado, Universidad de los llanos]. https://repositorio.unillanos.edu.co/handle/001/873

Moreno Castro, T. F. (2016). Emprendimiento y Plan de Negocios. Editores.

Rodríguez Moguel, E. (2005). Metodología de la Investigación. Universidad Juárez Autónoma de Tabasco.

Superintendencia de Compañías, Valores y Seguros del Ecuador. (2018). https://www.supercias.gob.ec/portalscrs/

Uribe Macías, M. (2013). Emprendimiento y empresarismo. Diferencias, conceptos, cultura emprendedora, idea y proyecto de empresa. Ediciones de la U.

Vallejo Chávez, L. (2016). Guía práctica de emprendimientos. La Caracola. 
Copyright (c) 2022 Carlos José Arias Ronquillo, Carolina Paola Jaramillo Bayas y Lorena Elizabeth Muquis Tituaña

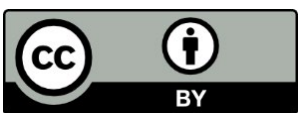

Este texto está protegido bajo una licencia internacional Creative Commons 4.0.

Usted es libre para Compartir - copiar y redistribuir el material en cualquier medio o formato - y Adaptar el documento - remezclar, transformar y crear a partir del material-para cualquier propósito, incluso para fines comerciales, siempre que cumpla las condiciones de Atribución. Usted debe dar crédito a la obra original de manera adecuada, proporcionar un enlace a la licencia, e indicar si se han realizado cambios. Puede hacerlo en cualquier forma razonable, pero no de forma tal que sugiera que tiene el apoyo del licenciante o lo recibe por el uso que hace de la obra.

$\underline{\text { Resumen de licencia - Texto completo de la licencia }}$ 